\title{
Partial Confirmation of a Conjecture on the Boxdot Translation in Modal Logic
}

\author{
Rohan French \\ School of Philosophy and Bioethics, \\ Monash University, \\ Victoria, 3800 \\ Australia \\ rohan.french@gmail.com \\ Lloyd Humberstone \\ School of Philosophy and Bioethics, \\ Monash University, \\ Victoria, 3800 \\ Australia \\ Lloyd.Humberstone@arts.monash.edu.au \\ Received by Greg Restall \\ Published July 29, 2009 \\ http://www .philosophy . unimelb.edu . au/ajl/2009 \\ (C) 2009 Rohan French and Lloyd Humberstone
}

\begin{abstract}
The purpose of the present note is to advertise an interesting conjecture concerning a well-known translation in modal logic, by confirming a (highly restricted) special case of the conjecture.
\end{abstract}

\section{THE CONJECTURE}

We work with the conventional language of (mono)modal logic, taking $\square$ and some functionally complete set of boolean connectives as primitive, to generate the set of formulas from the set of propositional variables, $p_{1}, \ldots, p_{n}, \ldots$ For $p_{1}$ and $p_{2}$ we generally write $p$ and $q$. Given a formula $A, \square A$ abbreviates the formula $\square A \wedge A$, and a formula in which all occurrences of $\square$ are in subformulas of the latter form will be called a $\square$-formula. The boxdot translation, $\tau_{\square}$, from modal formulas to modal formulas replaces all occurrences of $\square$ with $\square$. According to a well-known result, this translation embeds KT faithfully in (or 'into') $\mathrm{K}$ in the sense that for all formulas $A$ :

$$
\vdash_{K T} A \text { if and only if } \vdash_{K} \tau_{Ð}(A) .
$$


Because of its simplicity, the boxdot translation has been a traditional favourite in the philosophical literature on the significance of such embeddings, for example, $[7]$, [4] (and more briefly, by the same authors, in Example 2.9 of $[8]$ and $\$ 2$ of $[5]$. Recent technical studies in which it figures prominently include Goris [3] and Litak [6]; see also Zolin [Iㅇ, Lemma 5.9. ([3] and [IO] include references to the literature on modal provability logic, in which again $\square$ and $\tau_{\square}$ have enjoyed considerable currency.)

Now, experience with a considerable number of cases prompts the conjecture that the range of (normal: see below) modal logics in which $\tau_{\square}$ faithfully embeds KT-in the sense that reference to them can replace that to $\mathrm{K}$ in the biconditional inset above-comprises precisely the logics $S$ for which $\mathrm{K} \subseteq \mathrm{S} \subseteq \mathrm{KT} \Pi$ While it is not hard to see that $\tau_{\square}$ embeds $\mathrm{KT}$ faithfully into all the logics between $K$ and $K T$, and also (since $\vdash_{K T} A \leftrightarrow \tau_{\square}(A)$ for all $A$ ) that no proper extension of KT enjoys this status as a candidate 'target' for the embedding (with KT as 'source'), it is not so easy to see that only logics between $\mathrm{K}$ and $\mathrm{KT}$ are suitable candidates. (We have informally circulated this problem amongst several logicians with a special interest in modal translations, but none has so far been able to provide a solution.) What, in particular, is there to rule out the existence of a logic $\subseteq$-incomparable with $\mathrm{KT}$, in which $\mathrm{KT}$ is nonetheless embedded by $\tau_{\square}$ ? According to the conjecture just outlined, no such logic exists: $\tau_{\square}$ embeds KT only into sublogics of KT. "Logic", here, means normal modal logic 2 for these we use the labels of Chellas [I] where they exist, and in general for a set of formulas $\Gamma, S \oplus \Gamma$ is the smallest normal extension of $S$ containing all formulas in $\Gamma$. When $\Gamma=\{A\}$ for some formula $A$, we write "S $\oplus A$ " rather then "S$\oplus\{A\}$ ". In particular, then, $\mathrm{K} \oplus A$ is the smallest normal modal logic containing the formula $A$. In view of the facts about $\tau_{\square}$ cited above, the conjecture amounts to the conjecture that for any non-theorem $A$, of $K T$ ( $=$ $\mathrm{K} \oplus \mathrm{T}$, in the notation just introduced), there is a $\mathrm{K}$-unprovable $\square$-formula $\mathrm{B}$ with $\vdash_{K \oplus A}$ B. As we may put it, the normal extension of $K$ by any non-theorem

\footnotetext{
${ }^{\mathrm{I}}$ Considerations from the Kripke semantics point in a similar direction, but since these have proved suggestive rather than conclusive for us to date, they will not be in play explicitly in what follows. The idea is that whenever $A$ is not KT-provable, the reflexive closures of frames on which $A$ is valid will validate some formulas - the $A^{*}$ of the proof of Corollary 4 below-not valid on every reflexive frame. Note that we do not say that $A$ is valid on a frame if and only if $A^{*}$ is valid on its reflexive closure-by analogy with the well-known fact that $\tau_{\square}(A)$ is valid on a frame iff $A$ is valid on its reflexive closure. In fact, there can be no function $g(\cdot)$ from formulas to formulas with the property that for all formulas $A, A$ is valid on a frame iff $g(A)$ is valid on its reflexive closure, since different frames with the same reflexive closure need not validate the same formulas.

${ }^{2}$ We are taking a normal modal logic to be a set of formulas in the language described above, containing all the theorems of $\mathrm{K}$ and closed under Necessitation, Modus Ponens, and Uniform Substitution. For such a logic $S$ we write " $\vdash_{S} A$ " for " $A \in S$ " and read this as: " $A$ is provable in (or: is a theorem of) S". Because of the Uniform Substitution condition, it doesn't matter whether we think of KT as the smallest normal modal logic containing the formula $\square p \rightarrow p$ or as the smallest such logic containing all instances of the schema $\square A \rightarrow A$. Whereas Chellas [I] uses $T$ as the name of the schema, we use it as the name for the formula just mentioned.
} 
of KT is $\square$-nonconservative. (Note that the $\square$-formulas comprise precisely the image of the translation $\tau_{\varpi}$.)

Massaged into the form just given, the conjecture clearly admits of no counterexample in which $A$ is of modal degree $o$, since for such an $A \notin \mathrm{KT}, \mathrm{K} \oplus \mathrm{A}$ is the inconsistent logic (as $A$ is then a $\square$-free formula which is not a two-valued tautology). In the following section, we shall extend this to cover the case of formulas of modal degree $\mathrm{I}-$ formulas, that is, in which no occurrence $\square$ lies within the scope of another occurrence of $\square$. (On this convention a formula of modal degree $n$ is also of modal degree $n+1$.) Evidently this is a considerable limitation. The normal proper extensions of $\mathrm{K}$ axiomatizable by one-variable first degree formulas are precisely $K D, K D_{c}, K D$ !, $K T, K T_{c}, K T !, K \oplus \square \perp$ and the inconsistent logic. Amongst first-degree formulas considered as axioms by which to extend $\mathrm{K}$ without restriction as to the number of variables, the most famous are perhaps those from the infinite sequence of formulas Alt $_{n}(n \in \mathbb{N})$ from Segerberg [9], p. 52. This section concludes with some remarks on the general case, without any restriction as to modal degree.

Sometimes when $A \notin K T$, we can see that $K \oplus A$ is not $\square$-conservative because its theorems include $\tau_{\square}(A)$, and $\nvdash_{K} \tau_{\square}(A)$. Examples include the cases in which $A$ is $4(=\square p \rightarrow \square \square p)$ or $B(=p \rightarrow \square \diamond p$, where $\diamond p$ is $\neg \square \neg p)$, or $T_{c}$ ( $=p \rightarrow \square p)$. In other cases, $\nvdash_{K \oplus A} \tau_{\square}(A)$, such as when $A$ is $\square \perp, \square \perp \vee \diamond \square \perp$, or $D_{c}\left(=\diamond p \rightarrow \square p\right.$, a minor variant of Segerberg's Alt $\left.t_{1}\right)$; but here it is not hard to find $\square$-formulas other than $\tau_{\square}(A)$ which are not $K$-provable but which are of the form $\tau_{\varpi}$ (B) for KT-unprovable B. (In the cases just mentioned, take B as $\mathrm{T}_{\mathrm{c}}, \diamond(\square \mathrm{p} \vee \square \neg \mathrm{p})$, and $\mathrm{p} \vee \square(\mathrm{p} \rightarrow \mathrm{q}) \vee \square(\mathrm{p} \rightarrow \neg \mathrm{q})$, respectively.) The function $f$ described in the following section applies to any first degree $A$ to provide a $B$ with the desired behaviour.

\section{RESOLVING THE FIRST DEGREE CASE}

In any normal modal logic, a formula $A$ is provably equivalent to a conjunction of disjunctions of formulas of the form $\neg B \vee \neg \square C \vee \square D_{1} \vee \cdots \vee \square D_{n}$ in which $B$ is $\square$-free, and for the case of $A$ of modal degree $I$, the formulas $C, D_{1}, \ldots, D_{n}$ are also $\square$-free 3 we will write such 'basic disjunctions' in the implicational form:

$$
(\mathrm{B} \wedge \square \mathrm{C}) \rightarrow\left(\square \mathrm{D}_{1} \vee \cdots \vee \square \mathrm{D}_{\mathfrak{n}}\right),
$$

in which the consequent is understood as $\perp$ when $n=0$ and either or both of the conjuncts in the antecedent may be absent ${ }^{4}$ (If both are, we may think

${ }^{3}$ Fine $[2]$ is the locus classicus for normal forms in (normal) modal logic. Fine takes $\diamond$ as primitive rather than $\square$, and (essentially) works with disjunctive rather than (as here) conjunctive normal forms.

${ }^{4}$ We don't need to write " $\square C_{1} \wedge \ldots \wedge \square C_{m}$ " in the antecedent, since by normality this simplifies to $\square\left(C_{1} \wedge \cdots \wedge C_{m}\right)$. In terms of the disjunctive formulation, this is why we have just $\neg \square \mathrm{C}$ rather than $\neg \square \mathrm{C}_{1} \vee \cdots \vee \neg \square \mathrm{C}_{\mathrm{m}}$. 
of the antecedent as $T$ or, equivalently, identify the conditional with its consequent.)

Whether or not a given $A$ is a first degree modal formula, if $A$ is written as a conjunction $A_{1} \wedge \cdots \wedge A_{k}$ with each $A_{i}(1 \leqslant i \leqslant k)$ a basic formula (as inset above), we define $f(A)$ to be the conjunction of the formulas $f\left(A_{i}\right)$ where $f$ maps $A_{i}$, to the formula:

$$
(s \wedge B \wedge \boxminus C) \rightarrow\left(\sqcup\left(D_{1} \vee s\right) \vee \cdots \vee \sqcup\left(D_{n} \vee s\right)\right),
$$

in which $s$ is a propositional variable not occurring in $A_{i}$ (i.e. not occurring in the given $\left.B, C, D_{1}, \ldots, D_{n}\right)$, and the $\square$-notation is as explained in Section $\mathrm{r}$.

So defined, $f(A)$ is not unique, since various different conjunctive normal forms $\bigwedge_{i=1}^{k} A_{i}$ of $A$ can make a difference, as well as the choice of $s$ from the countable list of propositional variables (see the opening sentence of Section $\mathrm{I}$ ), though the first difference is inconsequential and the second could be ironed out by choosing $s$ for $A_{i}$ as the first $p_{j}$ in that enumeration which does not occur in $A_{i}$. Rather than making any such moves, however, we can simply take $f(A)$ to denote an arbitrarily selected formula satisfying the defining conditions.

LEMMA I For any formula $A: \vdash_{K} A \rightarrow f(A)$.

Proof: It suffices to observe that where $\bigwedge_{i=1}^{k} A_{i}$ is a normal form for $A$, we have $\vdash_{k} A_{i} \rightarrow f\left(A_{i}\right)$, for $1 \leqslant i \leqslant k$.

LEMma 2 For any formula A of modal degree $I$, if $\vdash_{K} f(A)$ then $\vdash_{K T} A$.

Proof: As in the proof of LemmaI, it suffices to check this for each $A_{i}$. Take $A_{i}$ as above, i.e., as $(B \wedge \square C) \rightarrow\left(\square D_{1} \vee \cdots \vee \square D_{n}\right)$, so that $f(A)$ is $(s \wedge B \wedge \square C) \rightarrow$ $\left(\odot\left(D_{1} \vee s\right) \vee \cdots \vee \boxminus\left(D_{n} \vee s\right)\right)$. Our hypothesis is that this is provable in $K$, so weakening the " $\square$ "s in the consequent to simple “ $\square$ "s, and unpacking the “ $\square$ " in the antecedent (together with some re-arranging), we have:

$$
\vdash_{K}((s \wedge B \wedge C) \wedge \square C) \rightarrow\left(\square\left(D_{1} \vee s\right) \vee \cdots \vee \square\left(D_{n} \vee s\right)\right)
$$

Since $A$, and therefore $A_{i}$, is of modal degree (at most) I, $s \wedge B \wedge C$ is a $\square$-free formula, so, using the fact that $\mathrm{K}$ is a (fully) modalized logic in the sense of [IOI, we can infer that either (a) $\vdash_{K}\left((s \wedge B \wedge C) \rightarrow \perp\right.$ or $(b) \vdash_{K} \square C \rightarrow\left(\square\left(D_{1} \vee s\right) \vee\right.$ $\cdots \vee \square\left(D_{n} \vee s\right)$ ). In case (a) we have $\vdash_{K} B \rightarrow \neg C$ (after substituting $B$ or $T$ for s), and therefore (since $\vdash_{K T} \square \mathrm{C} \rightarrow \mathrm{C}$ ) $\vdash_{\mathrm{KT}} \mathrm{B} \rightarrow \neg \square \mathrm{C}$, from which we conclude that $\vdash_{K T} A_{i}$. In case (b) we have, substituting $\bigwedge_{i=1}^{n} D_{i}$ for $s$ and simplifying: $\vdash_{K} \square \mathrm{C} \rightarrow\left(\square \mathrm{D}_{1} \vee \cdots \vee \square \mathrm{D}_{\mathrm{n}}\right)$, so $\vdash_{\mathrm{K}} A_{i}$ and therefore again $\vdash_{\mathrm{KT}} A_{i}$.

Putting these ingredients together settles the conjecture of Section I insofar as it bears on first degree formulas:

THEOREM 3 Suppose A is a first degree modal formula not provable in $\mathrm{KT}$. Then $\mathrm{K} \oplus \mathrm{A}$ proves some $\square$-formula not provable in $\mathrm{K}$. 
Proof: Let $A$ be as described. We may choose $f(A)$ as the desired $\square$-formula, since it is provable in $\mathrm{K} \oplus \mathrm{A}$, by Lemma $\mathrm{I}$ (and Modus Ponens), but not provable in $\mathrm{K}$, by Lemma 2 (contraposed).

For the first degree KT-unprovable formulas $A$ listed at the end of Section $I$, the formulas $B$ given there as having $B$ provable in $K \oplus A$ without $\tau_{\square}(B)$ provable in $K$ are minor variations on what the definition of $f(A)$ would deliver. For example, for the case of $A$ as $\square \perp$ we gave as a candidate $B$ the formula $p \rightarrow \square p$. Since this $A$ contains no propositional variables, taking $s$ as the first variable not occurring in $A$ means that $s$ is $p$ (alias $p_{1}$ ), and $f(A)$ is therefore $p \rightarrow \boxminus(\perp \vee p)$, which is provably equivalent in $K$ to $p \rightarrow \square p$. More generally, since we may have to make substitutions, we can say for the proffered examples of $B$ that $B$ and $f(A)$ are $K$-interducible in the sense that $K \oplus B=K \oplus f(A)$.5 This happens in the case of $A=p \rightarrow \square p$ in which again we gave $B$ as $p \rightarrow \square p$, while $f(A)$ is $(p \wedge q) \rightarrow \boxminus(p \vee q)$ and we need to substitute $p$ for $q$ before proceeding further.

Returning to the conjecture as first formulated in Section I, we have the following:

COROLLARY 4 If $S=K \oplus \Gamma$ for a set $\Gamma$ of first degree formulas and $S \nsubseteq K T$, then $\tau_{\square}$ does not embed $K T$ faithfully in $S$.

Proof: Given that $\mathrm{S} \nsubseteq \mathrm{KT}$, pick $A \in \Gamma \backslash \mathrm{KT}$, and the proof of Theorem 3 shows that $f(A)$ is a $\square$-formula in $K \oplus A \backslash K$. Thus we have (I) $\nvdash_{K} f(A)$ while (2) $\vdash_{S} f(A)$. Since $f(A)$ is a $\square$-formula, replacing every $\square$ in $f(A)$ with $\square$ gives a formula $A^{*}$ for which $f(A)=\tau_{\odot}\left(A^{*}\right)$, and (I) and (2) can be reformulated as $\left(1^{\prime}\right): \nvdash_{K} \tau_{\square}\left(A^{*}\right),\left(2^{\prime}\right): \vdash_{S} \tau_{\square}\left(A^{*}\right)$. From the fact (recalled in Section I) that $\tau_{\square}$ embeds $K T$ in $K$, we infer from $\left(1^{\prime}\right)$ that $\nvdash_{K T} A^{*}$. If it were also the case that $\tau_{\square}$ faithfully embedded $K T$ in $S,\left(2^{\prime}\right)$ would imply that $\vdash_{K T} A^{*}$ : a contradiction.

What is wanted, then, is a way of lifting the restriction to first degree formulas from Theorem 3 (and Corollary 4) - or else of showing, with a counterexample, that it cannot be lifted. Should the former turn out to be possible, perhaps the proof of Theorem 3 can be turned into the inductive step of a proof of the desired general result (by induction on the modal degree of formulas); however, we do not currently see how the details of such an argument would go.

\footnotetext{
${ }^{5}$ Indeed, since the rule of necessitation is not required here, we could say more informatively that $K+B=K+f(A)$, where $S+\Gamma$ is the smallest (not necessarily normal) modal logic extending $\mathrm{S}$ and containing all formulas in $\Gamma$. In fact, everything said in Lemma 2 about KT applies to the quasi-normal modal logic $\mathrm{K}+\mathrm{T}\left(=\mathrm{K}[\mathrm{T}]^{0}\right.$ from [9], p. I79), suggesting a variant on Corollary 4 below in which the references to $K \oplus \Gamma$ and $K T$ are replaced by references to $K+\Gamma$ and $K+T$, the qualification "first degree" is dropped, and reference to $\tau_{\square}$ is replaced by a one to the translation $\tau_{0}$, where $\tau_{0}(A)$ is the result of replacing only the outermost (i.e., not modally embedded) occurrences of $\square$ in $A$ by $\square$. We do not go into this in any detail because of the marginal interest of $\tau_{0}$ by comparison with $\tau_{\square}$.
} 


\section{REFERENCES}

[I] B. F. Chellas, Modal Logic: An Introduction, Cambridge University Press, Cambridge 1980.

[2] Kit Fine, 'Normal Forms in Modal Logic', Notre Dame fournal of Formal Logic I6 (I975), 229-237.

[3] E. Goris, 'Interpreting Knowledge into Belief in the Presence of Negative Introspection', Technical Report TR-2007005, CUNY Ph.D. Program in Computer Science, 2007.

[4] Lloyd Humberstone, 'Béziau's Translation Paradox', Theoria 7r (2005), I38I8I.

[5] Lloyd Humberstone, 'Weaker-to-Stronger Translational Embeddings in Modal Logic', pp. 279-297 in G. Governatori, I. Hodkinson and Y. Venema, (eds.) Advances in Modal Logic, Vol. 6, College Publications, London 2006.

[6] Tadeusz Litak, 'The Non-reflexive Counterpart of Grz', Bulletin of the Section of Logic 36 (2007), 195-208.

[7] F. J. Pelletier, 'Six Problems in "Translational Equivalence", Logique et Analyse 27 (1984), 423-434.

[8] F. J. Pelletier and A. Urquhart, 'Synonymous Logics', fournal of Philosophical Logic 32 (2003) 259-285; Correction: ibid. 37 (2008), 95-100.

[9] Krister Segerberg, An Essay in Classical Modal Logic, Filosofiska Studier, Uppsala I97I.

[ı] E. E. Zolin, 'Embeddings of Propositional Monomodal Logics', Logic fournal of the IGPL 8 (2000), 86I-882. 
The Australasian fournal of Logic (ISSN I448-5052) disseminates articles that significantly advance the study of logic, in its mathematical, philosophical or computational guises. The scope of the journal includes all areas of logic, both pure and applied to topics in philosophy, mathematics, computation, linguistics and the other sciences.

Articles appearing in the journal have been carefully and critically refereed under the responsibility of members of the Editorial Board. Only papers judged to be both significant and excellent are accepted for publication.

The journal is freely available at the journal website at

$$
\text { http://www.philosophy.unimelb.edu.au/ajl/ }
$$

All issues of the journal are archived electronically at the journal website.

SuBSCRIPTIONS Individuals may subscribe to the journal by sending an email, including a full name, an institutional affiliation and an email address to the managing editor at ajl-editors@unimelb.edu.au Subscribers will receive email abstracts of accepted papers to an address of their choice. For institutional subscription, please email the managing editor at ajl-editors@unimelb.edu.au

Complete published papers may be downloaded at the journal's website at http: //www.philosophy.unimelb.edu.au/ajl/ The journal currently publishes in pdf format.

Submission The journal accepts submissions of papers electronically. To submit an article for publication, send the $\mathrm{LT}_{\mathrm{E}} \mathrm{X}$ source of a submission to a member of the editorial board. For a current list of the editorial board, consult the website.

The copyright of each article remains with the author or authors of that article. 